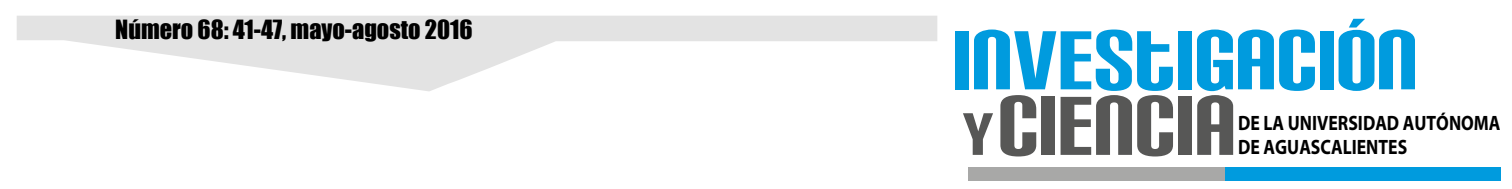

\title{
Ecuaciones de volumen para Arbutus spp. (madroño) en la región de Pueblo Nuevo, Durango
}

\author{
Volume table for Arbutus spp. (madrono) in the region of Pueblo \\ Nuevo, Durango
}

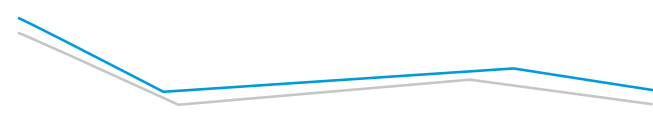

Francisco Cruz Cobos ${ }^{1 *}$, Roberto Mendía Santana', Abner Alain Jiménez Flores', Juan Abel Nájera Luna', Francisco Cruz García ${ }^{2}$

\begin{abstract}
Cruz Cobos, F., Mendía Santana, R., Jiménez Flores, A. A., Nájera Luna, J. A. Cruz García, F. Ecuaciones de volumen para Arbutus spp. (madroño) en la región de Pueblo Nuevo, Durango. Investigación y Ciencia de la Universidad Autónoma de Aguascalientes. Número 68: 41-47 mayo-agosto 2016
\end{abstract}

\section{RESUMEN}

El objetivo del trabajo fue ajustar modelos para estimar el volumen total de Arbutus spp. (madroño) en la región de Pueblo Nuevo, Durango. Se derribaron 120 árboles de diferentes categorías diamétricas, se midieron las variables diámetro normal (D), altura total (H), diámetro de la base (DB), diámetro de copa (DC) y el volumen de cada árbol (V). Se probaron 20 modelos de una entrada y 15 de dos o más entradas, el ajuste de las ecuaciones se hizo por mínimos cuadrados ordinarios y mínimos cuadrados ponderados. Para la selección del mejor modelo se tomó en cuenta, el RCME, $R^{2}$ y el nivel de significancia de los parámetros. El mejor modelo de una entrada fue el M2 (alométrico de Berkhout), que considera como variable independiente el diámetro normal; el de dos entradas, M21 (Schumacher-Hall), que considera como variables independientes el diámetro normal y la altura total.

\section{ABSTRACT}

Mathematical equations were fitted to estimate the total volume in madrono trees (Arbutus spp.),

Palabras clave: modelos de volumen, relaciones alométricas, madroño.

Keywords: volume models, allometric relations, madrono.

\section{Recibido: 3 de marzo de 2016, aceptado: 13 de mayo de 2016}

Instituto Tecnológico de El Salto.

Programa de Doctorado en Manejo de Vida Silvestre y Desarrollo Sustentable, Universidad Autónoma de Nuevo León.

* Autor para correspondencia: cobos_cruz@yahoo.com.mx in the region of Pueblo Nuevo, Durango. 120 trees of different diameter categories were demolished, the variables normal diameter (D), total height $(H)$, diameter of the base (DB) and crown diameter (DC) were measured. The volume of each tree was obtained with the xylometer. 20 models of one input and 15 of two or more inputs were tested for to predict the volume, the equations adjustment was by Ordinary Least Squares and Weighted Least Squares. For the selection of the best model, the RCME, $R^{2}$ and the level of significance of the parameters were taken into account. The best model of one input was the M2 (allometric of Berkhout), which considers the independent variable normal diameter, the two inputs M21 (Schumacher-Hall), that consider independent variables the normal diameter and total height.

\section{INTRODUCCIÓN}

Una de las variables que es indispensable conocer en estudios de inventario de manejo para llevar a cabo un aprovechamiento forestal maderable es el volumen de los árboles en pie. Lo anterior se logra mediante la medición de algunas variables como el diámetro y la altura, para luego estimar el espesor de cada árbol y posteriormente extrapolar la información a todo un rodal (Acosta y Carrillo, 2008). Al grupo de ecuaciones que nos permite estimar el volumen en pie se le denomina Sistema Volumétrico o de Cubicación. La obtención de un sistema adecuado permitirá obtener un inventario maderable confiable y servirá como herramienta para el buen manejo de los recursos forestales. Las tablas de volumen son una herramienta muy útil para un estricto control de los aprovechamientos maderables, esto facilita la ejecución del manejo sustentable de los bosques. Con el uso cotidiano de estas tablas, los prestadores 
de servicios técnicos forestales estiman la dimensión de la madera de los árboles de cada cosecha anual, simplemente midiendo el diámetro y la altura de los árboles (Acosta y Carrillo, 2008). En la región de Pueblo Nuevo, Durango, se han hecho estudios y tablas de volumen, la mayoría de ellos en especies de coníferas; pero para Arbutus spp. no se tiene algún antecedente en estudios de estimación del mismo. El objetivo del presente trabajo fue ajustar modelos de una y dos o más entradas que permitan estimar el volumen total con corteza de este género y generar herramientas matemáticas que faciliten su evaluación.

\section{MATERIALES Y METODOS}

El proyecto se realizó en la UMAFOR 1008 El Salto, específicamente en los ejidos La Victoria, San Esteban y La Campana, estos se ubican en la provincia fisiográfica Sierra Madre Occidental, en la subprovincia Gran Meseta y cañones duranguenses (Figura 1). La altura promedio en los ejidos es 2,200 a 2,500 m.s.n.m. En cuanto a los climas que se presentan en la región empleando la clasificación de climas de Köeppen modificada por García (1973) se ubican el C(E) (W2): clima semifrío subhúmedo con lluvias en verano y C(W2): clima templado subhúmedo. Las comunidades vegetales más importantes de la región están compuestas por bosques mezclados con especies del género Pinus y Quercus.

Se muestrearon un total de 120 árboles de cinco especies de Arbutus y se abarcaron la mayoría

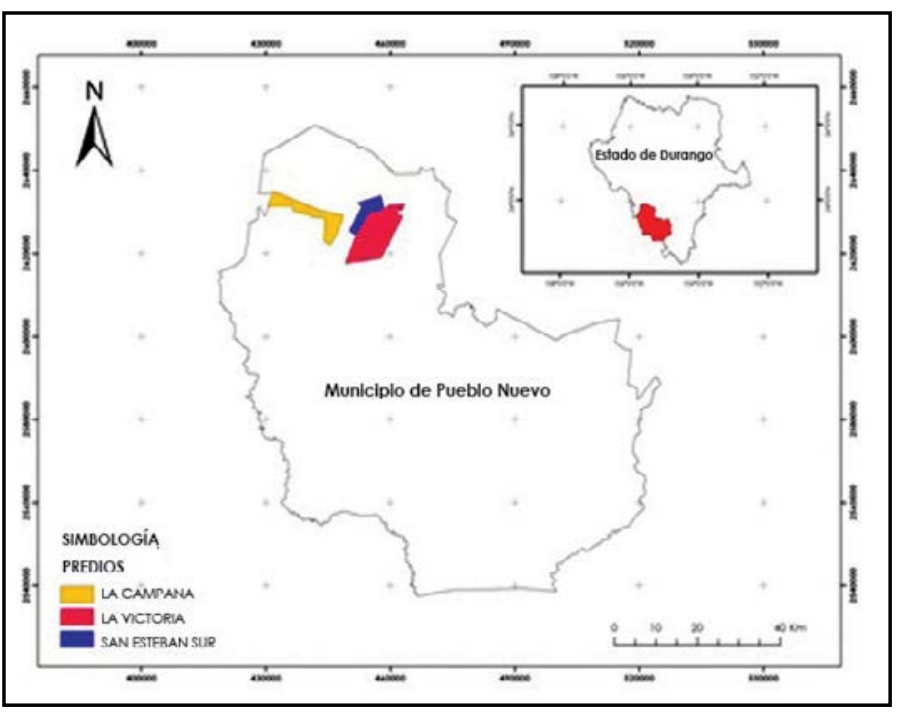

Figura 1. Localización del área de estudio. Elaboración propia.

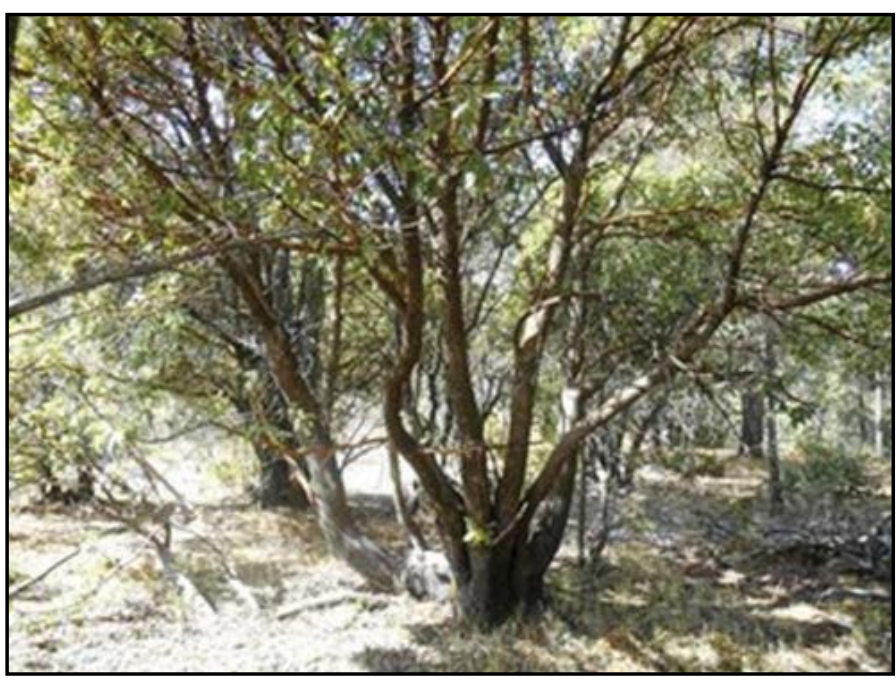

Figura 2. Características del fuste del madroño. Fotografía tomada por Francisco Cruz Cobos.

de las categorías diamétricas, clases sociológicas, diferentes condiciones topográficas y edáficas.

A cada árbol muestreado se le midieron las variables: diámetro de la base del tallo $(\mathrm{cm})$, diámetro normal con corteza a $1.30(\mathrm{~m})$, diámetro de copa total por tallo $(m)$, altura total por tallo $(m)$, volumen total del árbol $\left(\mathrm{m}^{3}\right)$. Estos árboles en algunos casos crecen formando cepas con dos o más fustes y bifurcaciones, en tales situaciones la medición de las variables se hizo de la siguiente manera: los fustes con bifurcación antes de los $30 \mathrm{~cm}$ del suelo se consideraron como dos árboles, los fustes bifurcados por arriba de $30 \mathrm{~cm}$ y por debajo de $130 \mathrm{~cm}$ se consideraron como un árbol y se obtuvo el diámetro normal como el promedio de los dos diámetros; de igual manera se hizo la operación para el diámetro de copa y la altura y los árboles que presentaron bifurcación después de $130 \mathrm{~cm}$ se consideraron como un solo tallo (Figura 2). El cálculo del volumen de cada árbol se realizó mediante el xilómetro.

Para la estimación del volumen total, se ajustaron modelos volumétricos de una entrada (Tabla 1) y de dos y tres entradas (Tabla 2).

Para la evaluación del ajuste de los modelos se utilizó el análisis gráfico y numérico, a través del mayor valor de coeficiente de determinación ajustado $\left(R^{2}\right)$, el menor valor de la raíz del error medio cuadrático (RCME), las representaciones gráficas y el nivel de significancia de los parámetros. 


\section{InVESEIGACIÓn

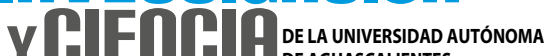

Tabla 1. Modelos de volumen de una entrada ajustados

\begin{tabular}{|c|c|}
\hline Modelo & Expresión \\
\hline M1 & $V=\beta_{0}+\beta_{1} *(D)^{2}$ \\
\hline M2 & $V=\beta_{0} *(D)^{\beta_{1}}$ \\
\hline M3 & $V=\beta_{0} * D+\beta_{1} *(D)^{2}$ \\
\hline M4 & $V=\beta_{0}+\beta_{1} * D+\beta_{2} *(D)^{2}$ \\
\hline M5 & $V=\beta_{0}+\beta_{1} * D^{2}+\beta_{2} *(D)^{3}$ \\
\hline M6 & $V=\beta_{0}+\beta_{1} * D+\beta_{2} *(D)^{2}+\beta_{3} *(D)^{3}$ \\
\hline M7 & $V=\beta_{0}+\beta_{1} *(D B)^{2}$ \\
\hline M8 & $V=\beta_{0} *(D B)^{\beta_{1}}$ \\
\hline M9 & $V=\beta_{0} * D B+\beta_{1} *(D B)^{2}$ \\
\hline M10 & $V=\beta_{0}+\beta_{1} * D B+\beta_{2} *(D B)^{2}$ \\
\hline M11 & $V=\beta_{0}+\beta_{1} * D B^{2}+\beta_{2} *(D B)^{3}$ \\
\hline M12 & $V=\beta_{0}+\beta_{1} * D B+\beta_{2} *(D B)^{2}+\beta_{3} *(D B)^{3}$ \\
\hline M13 & $V=\beta_{0}+\beta_{1} *(H)^{2}$ \\
\hline M14 & $V=\beta_{0} *(H)^{\beta_{1}}$ \\
\hline M15 & $V=\beta_{0} * H+\beta_{1} *(H)^{2}$ \\
\hline M16 & $V=\beta_{0}+\beta_{1} * H+\beta_{2} *(H)^{2}$ \\
\hline M17 & $V=\beta_{0}+\beta_{1} * H^{2}+\beta_{2} *(H)^{3}$ \\
\hline M18 & $V=\beta_{0}+\beta_{1} *(D C)^{2}$ \\
\hline M19 & $V=\beta_{0} *(D C)^{\beta_{1}}$ \\
\hline M20 & $V=\beta_{0} * D C+\beta_{1} *(D C)^{2}$ \\
\hline
\end{tabular}

Elaboración propia.

$$
\begin{gathered}
R^{2}=1-(n-1) \sum_{i=1}^{n} \frac{\left(Y_{i}-\widehat{Y}_{i}\right)^{2}}{n-p} / \sum_{i=1}^{n}\left(y_{i-} \bar{y}_{i}\right)^{2} \\
R C M E=\sqrt{\frac{\sum_{i=1}^{n}\left(Y_{i}-\widehat{Y}_{i}\right)^{2}}{n-p}}
\end{gathered}
$$

donde: $Y_{i}, \widehat{\boldsymbol{Y}}_{\boldsymbol{i}}$ y $\bar{Y}_{i}$ son valores observados, estimados y media del volumen; $n y p$ son el número de observaciones y de parámetros, respectivamente.

Torres y Magaña (2001) mencionan que es común que la mayor parte de los modelos de volumen sufran problemas de heterocedasticidad, dado que a mayor valor de diámetro y altura la variación en el volumen de los árboles se hace más grande, por lo que es necesaria esta corrección con el uso de mínimos cuadrados ponderados. Una vez corregido con este procedimiento se obtienen estimadores insesgados y eficientes (Gujarati, 1988).

El análisis gráfico de los residuos estudentizados frente a los valores estimados con los modelos selec-
Tabla 2. Modelos de volumen de dos y tres entradas ajustados

\begin{tabular}{|l|l|}
\hline Modelo & \multicolumn{1}{|c|}{ Expresión } \\
\hline M21 & $V=\beta_{0} *(D)^{\beta_{1} *(H)^{\beta_{2}}}$ \\
\hline M22 & $V=\beta_{0} *(D)^{2} * H$ \\
\hline M23 & $V=\beta_{0} *(D * H)^{\beta_{1}}$ \\
\hline M24 & $V=\beta_{0}+\beta_{1} *(D)^{2} * H$ \\
\hline M25 & $V=\beta_{0}+\beta_{1} * H+\beta_{2} *(D)^{2} * H$ \\
\hline M26 & $V=\beta_{0}+\beta_{1} *(D)^{2}+\beta_{2} *(D)^{2} * H+\beta_{3} * H$ \\
\hline M27 & $V=(D)^{2} / \beta_{0}+\beta_{1} / H$ \\
\hline M28 & $V=\beta_{0}+\beta_{1} *(D)^{\beta_{2}} *(H)^{\beta_{3}}$ \\
\hline M29 & $V=E x p\left(\beta_{0}+\beta_{1} * H+\beta_{2} * D B\right.$ \\
\hline M30 & $V=E x p\left(\beta_{0}+\beta_{1} * H+\beta_{2} * D B+\beta_{3} * D C\right.$ \\
\hline M31 & $V=\beta_{0}+\beta_{1} *(D B)^{2} * H+\beta_{2} * D C$ \\
\hline M32 & $V=\beta_{0}+\beta_{1} *(D B)^{2} * H$ \\
\hline M33 & $V=\beta_{0}+\beta_{1} *(D)^{2} * H$ \\
\hline M34 & $V=\beta_{0}+\beta_{1} * D+\beta_{2} * D * H+\beta_{3} *(D)^{2}+\beta_{4} *(D)^{2} * H$ \\
\hline M35 & $V=\beta_{0}+\beta_{1} * D+\beta_{2} * D * H+\beta_{3} *(D)^{2}+\beta_{4} * H+\beta_{5} *(D)^{2} * H$ \\
\hline$V$
\end{tabular}

$\mathrm{V}=$ volumen $\left(\mathrm{m}^{3}\right), \mathrm{D}=$ diámetro normal $(\mathrm{cm}), \mathrm{H}=$ altura total $(\mathrm{m}), \mathrm{DB}=$ diámetro de base $(\mathrm{cm}), \mathrm{DC}=$ diámetro de copa $(\mathrm{m})$ y $\beta_{0}, \beta_{1}, \beta_{2}, \beta_{3}, \beta_{4}, \beta_{5}=$ parámetros a ser estimados. Elaboración propia.

cionados mostró problemas de heterocedasticidad, por lo que se hizo la corrección a través de regresión ponderada, que consiste en asignar una variable de peso igual a la inversa de cada observación. Dicha varianza $\sigma_{\mathrm{i}}{ }^{2}$, que es desconocida, se estimó utilizando una función exponencial $\sigma_{\mathrm{i}}{ }^{2}=\gamma \cdot D^{k}$. Los valores de $\gamma$ y del exponente $k$ se optimizaron empleando la metodología propuesta por Harvey (1976), que consiste en emplear los errores del modelo ajustado sin pesos como variable dependiente en el modelo potencial de varianza del error; finalmente, el peso considerado fue $1 / D^{k}$.

\section{RESULTADOS}

Las principales estadísticas descriptivas de las variables utilizadas muestran que se cubrió un amplio rango de alturas $(\mathrm{H})$ y diámetros normales (D). Sin embargo, por la poca abundancia mostrada en cuatro de las cinco especies analizadas en el área de estudio (Tabla 3), el tamaño de muestra no se consideró suficiente para hacer un ajuste de los modelos por especie como lo recomienda Torres y Magaña (2001), de muestrear al menos 50 árboles o 10 por categoría diamétrica, por lo que 
las ecuaciones se ajustaron a nivel de género. Se observaron además de manera gráfica las similitudes de los volúmenes con respecto a los diámetros entre las diferentes especies (Figura 3).

Tabla 3. Estadísticos descriptivos de la muestra empleada por especie

\begin{tabular}{|c|l|c|c|c|c|c|c|}
\hline \multirow{2}{*}{ No. árboles } & \multirow{2}{*}{ Especie } & \multicolumn{2}{|c|}{$\mathbf{D}(\mathbf{c m})$} & \multicolumn{2}{c|}{$\mathbf{H}(\mathrm{m})$} & \multicolumn{2}{c|}{$\mathbf{V}\left(\mathbf{m}^{3}\right)$} \\
\cline { 3 - 8 } & & Mín & Máx & Mín & Máx & Mín & Máx \\
\hline 65 & A. bicolor & 5.9 & 36 & 4.6 & 14.5 & 0.0065 & 0.8097 \\
\hline 25 & A. tessellata & 5.5 & 25 & 3.8 & 12.72 & 0.0073 & 0.2766 \\
\hline 4 & A. arizonica & 9.5 & 24 & 5.57 & 10.85 & 0.0324 & 0.3553 \\
\hline 16 & A. madrensis & 7.5 & 22.25 & 3.3 & 11.85 & 0.0122 & 0.1994 \\
\hline 10 & A. xalapensis & 9.2 & 20.5 & 5.2 & 8.9 & 0.0222 & 0.2606 \\
\hline
\end{tabular}

Elaboración propia.

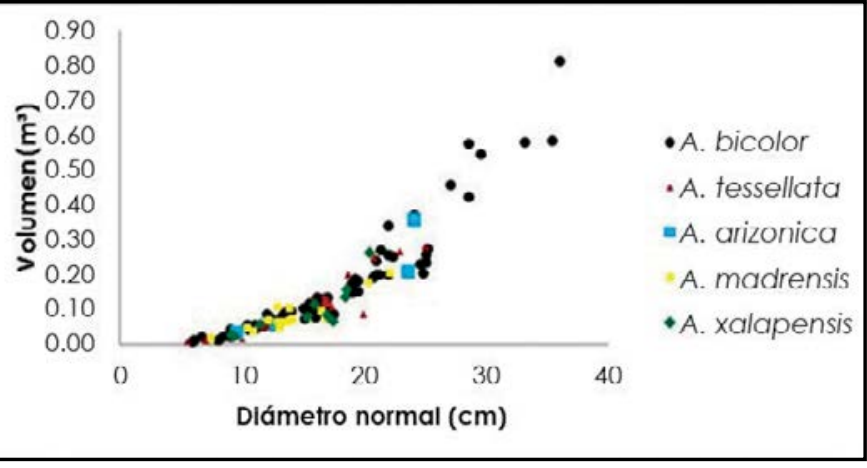

Figura 3. Tendencia del volumen observado vs. diámetro normal por especie. Elaboración propia.

Tabla 4. Estadísticos de ajuste de las ecuaciones volumétricas de una entrada

\begin{tabular}{|c|c|c|c|c|c|}
\hline Modelo & $\boldsymbol{R}^{\mathbf{2}}$ & $\boldsymbol{R C M E}$ & Modelo & $\boldsymbol{R}^{\mathbf{2}}$ & RCME \\
\hline M1 & 0.919 & 0.039 & M13 & 0.543 & 0.1134 \\
\hline M2 & 0.925 & 0.0375 & M14 & 0.538 & 0.1141 \\
\hline M3 & 0.924 & 0.0379 & M15 & 0.545 & 0.1132 \\
\hline M4 & 0.926 & 0.0376 & M16 & 0.545 & 0.1141 \\
\hline M5 & 0.925 & 0.0378 & $M 17$ & 0.544 & 0.1143 \\
\hline M6 & 0.926 & 0.0378 & $M 18$ & 0.308 & 0.1397 \\
\hline M7 & 0.874 & 0.0595 & $M 19$ & 0.369 & 0.1334 \\
\hline M8 & 0.894 & 0.0545 & $M 20$ & 0.351 & 0.1353 \\
\hline M9 & 0.884 & 0.0571 & & & \\
\hline M10 & 0.891 & 0.0557 & & & \\
\hline M11 & 0.896 & 0.0545 & & & \\
\hline M12 & 0.8981 & 0.0544 & & & \\
\hline
\end{tabular}

Todos los parámetros fueron significativos al $5 \%$.

Elaboración propia.
En la Tabla 4 se muestran los estadísticos de ajuste de los modelos de volumen total con corteza de una entrada, en donde se encontraron los mejores ajustes con los modelos Ml-M6 que consideran como variable independiente el diámetro normal. Tanto los estadísticos de ajuste de estos modelos como su representación gráfica (Figura 4) son muy similares, por lo que se seleccionó el modelo M2 por su simplicidad en la implementación.

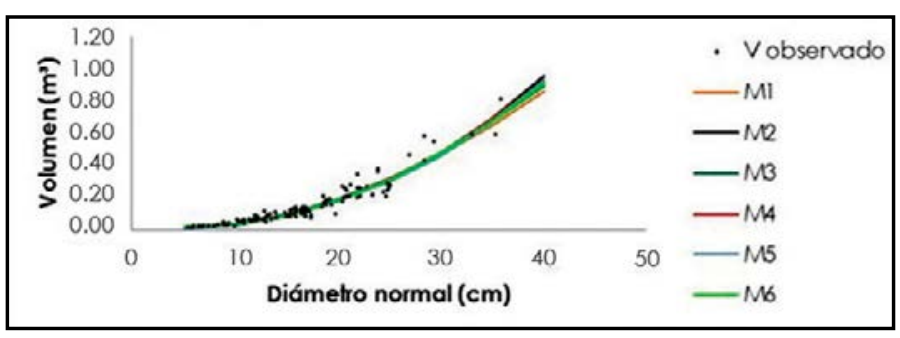

Figura 4. Volumen observado y estimado a partir del diámetro normal. Elaboración propia.

Una vez corregida la heterocedasticidad (Figura 5), la expresión final del modelo M2 se presenta en la Tabla 5, de manera que la estructura queda conformada así:

$$
V=0.000144 * D^{2.379922}
$$

Tabla 5. Estadísticos finales de ajuste de la ecuación M2 con mínimos cuadrados ponderados

\begin{tabular}{|c|c|c|c|c|}
\hline Modelo & $\boldsymbol{R}^{\mathbf{2}}$ & RCME & $\boldsymbol{\beta 0}$ & $\boldsymbol{\beta 1}$ \\
\hline M2 & 0.9244 & 0.0378 & 0.000144 & 2.379922 \\
\hline
\end{tabular}

Elaboración propia. 


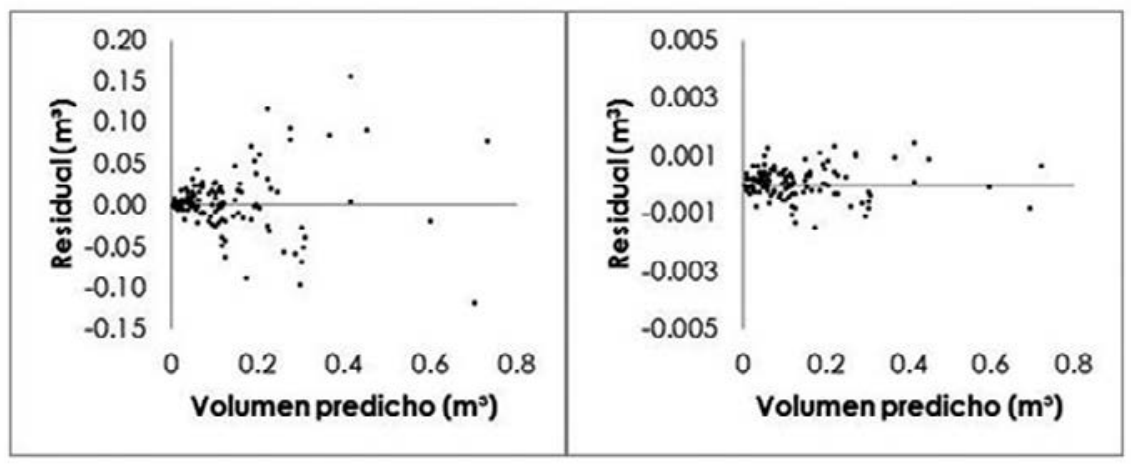

Figura 5. Valores predichos vs. residuos estudentizados con evidencia de problemas de heterocedasticidad (izquierda) y los obtenidos con regresión ponderada (derecha). Elaboración propia.

Todos los modelos probados presentan buenos ajustes a excepción de los modelos M31 y M32 (Tabla 6).

Una vez corregida la heterocedasticidad por mínimos cuadrados ponderados (Figura 6), la expresión final del modelo M21 se presenta en la Tabla 7, de modo que la estructura queda de la siguiente forma:

Tabla 6. Estadísticos de ajuste para las ecuaciones volumétricas de dos o más entradas

\begin{tabular}{|c|c|c|c|c|c|}
\hline Modelo & $\boldsymbol{R}^{\mathbf{2}}$ & $\boldsymbol{R C M E}$ & Modelo & $\boldsymbol{R}^{\mathbf{2}}$ & $\boldsymbol{R}$ CME \\
\hline M21 & 0.955 & 0.0009 & M29 & 0.916 & 0.0016 \\
\hline M22 & 0.954 & 0.0009 & $M 30$ & 0.930 & 0.0014 \\
\hline M23 & 0.928 & 0.0014 & M31 & 0.896 & 0.0020 \\
\hline M24 & 0.954 & 0.0009 & $M 32$ & 0.895 & 0.0020 \\
\hline M26 & 0.956 & 0.0008 & $M 33$ & 0.954 & 0.0009 \\
\hline M27 & 0.956 & 0.0009 & $M 34$ & 0.956 & 0.0009 \\
\hline M28 & 0.920 & 0.0015 & $M 35$ & 0.956 & 0.0009 \\
\hline
\end{tabular}

Todos los parámetros fueron significativos al 5\%, con excepción de los modelos M34 y M35. Elaboración propio.

Tabla 7. Estadísticos finales del ajuste de la ecuación M21 por mínimos cuadrados ponderados

\begin{tabular}{|c|c|c|c|c|c|}
\hline Modelo & $\boldsymbol{R}^{\mathbf{2}}$ & $\boldsymbol{R C M E}$ & $\boldsymbol{\beta 0}$ & $\boldsymbol{\beta} 1$ & $\boldsymbol{\beta 2}$ \\
\hline M21 & 0.9453 & 0.0321 & 0.000116 & 2.078374 & 0.501168 \\
\hline
\end{tabular}

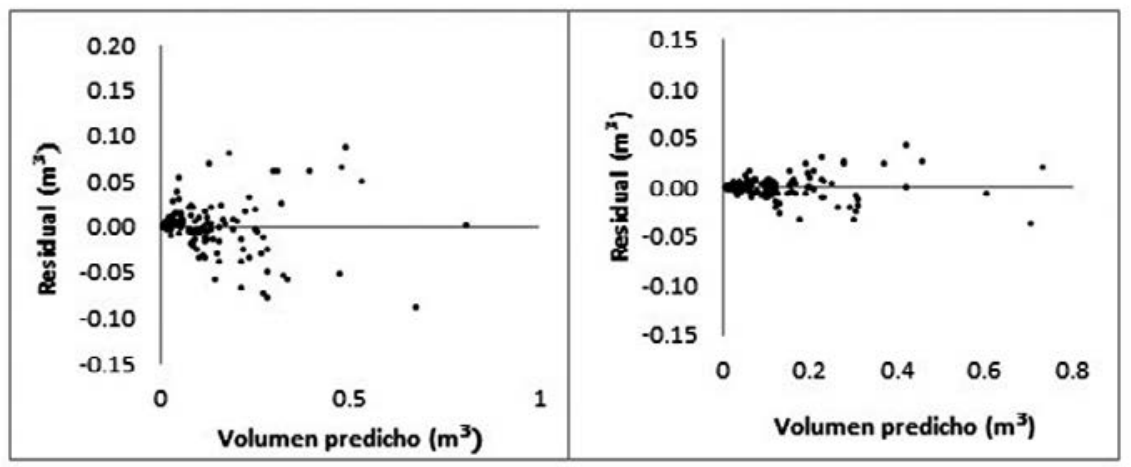

Figura 6. Valores predichos vs. residuos estudentizados con evidencia de problemas de heterocedasticidad (izquierda) y los obtenidos con regresión ponderada (derecha). Elaboración propia. 


\section{DISCUSIÓN}

Se puede observar que se cubrió un amplio rango en diámetros y alturas (Tabla 3). Las mayores dimensiones de estas variables se encontraron en Arbutus bicolor, seguidos de A. tessellata, A. arizonica, A. madrensis y A. xalapensis, también se observa que A. bicolor fue la especie más abundante y la menos abundante fue A. arizonica, debido a que su rango de distribución geográfica y abundancia disminuye con la altitud (González et al., 2012a; De León et al., 2013).

Los mejores ajustes para las ecuaciones de una entrada se obtuvieron utilizando como variable independiente el diámetro normal (M1-M6), seguidos con ajustes aceptables los modelos que consideran la variable diámetro de base (M7-M12) (Tabla 4); mientras que los ajustes más pobres se obtuvieron con los modelos que consideran la altura total y el diámetro de copa, esto en parte es debido a que la altura total es influenciada y varía por la calidad de sitio y el diámetro de copa se ve afectado a causa de que este género en la mayoría de los casos se desarrolla formando cepas de dos o más tallos (González et al., 2012b). El modelo M2 (alométrico de Berkhout) fue recomendado por Bustillos (2012) en un estudio para Pinus douglasiana Martínez y P. oocarpa Schiede en la UMAFOR 1008 El Salto, Durango.

Todos los modelos de dos entradas presentaron buenos ajustes a excepción de los modelos M31 y M32 (Tabla 6), que consideran como variables independientes el diámetro de la base (DB), la altura total $(\mathrm{H})$ y el diámetro de copa (DC), por lo que se seleccionó el modelo M21 (Schumacher-Hall) por su simplicidad en la implementación, ya que tiene como variables independientes el diámetro normal y la altura total. De la misma manera, Reyes Valdovinos (2006) en un estudió para Eucalyptus grandis y E. urophylla en los límites de Veracruz y Oaxaca, ajustó varios modelos y concluyó que el modelo M21 (Schumacher-Hall) era el mejor para la estimación de volumen total, este modelo es ampliamente usado para la estimación del volumen total de coníferas de la región de Pueblo Nuevo, Durango (Vargas et al., 2012), lo que afirma la flexibilidad para modelar también en especies latifoliadas.

\section{CONCLUSIONES}

El mejor ajuste en modelos de una entrada se obtuvo cuando se utilizó el diámetro normal como variable independiente, por lo que se recomienda el modelo M2 (alométrico de Berkhout) por su simplicidad en la implementación.

El mejor ajuste para la estimación de volumen para los modelos de dos o más entradas se obtuvo con la ecuación M21 (Schumacher-Hall, alométrica), esta ecuación considera el diámetro normal y la altura total como variables independientes.

Los modelos propuestos son precisos y permitirán hacer estimaciones confiables del volumen en pie de este importante género en la región de Pueblo Nuevo. 
LITERATURA CITADA

- $\quad$ Acosta, M. M. y CARRILLO, A. F. Tabla de volumen total con y sin corteza para Pinus montezumae Lamb. en el Estado de Hidalgo. Folleto técnico No. 7. Pachuca, Hidalgo, México: INIFAP Campo Experimental Pachuca, 20 pp., 2008.

- BUSTILLOS, A. E. G. Sistema volumétrico para Pinus douglasiana Martínez y Pinus oocarpa Schiede en la UMAFOR 1008 El Salto. Tesis profesional. El Salto, Pueblo Nuevo, Durango: Instituto Tecnológico de El Salto, 54 pp., 2012.

- DE LEÓN, M. G. D. et al. Distribución de la vegetación a través de un transecto sobre la Sierra Madre Occidental de Durango, México. Revista Latinoamericana de Recursos Naturales, 9(1): 30-40, 2013.

- García, M. E. Modificación al sistema de clasificación climática de Köppen (para adaptarlo a las condiciones de la República Mexicana). D. F., México: UNAM. Instituto de Geografía, 246 pp., 1973.

- GONZÁLEZ, E. M. S. et al. Delimitación de taxonomía de Arbutus mollis y A. occidentalis (Ericaceae). Acta Botánica Mexicana, $100,49-81,2012 a$
- GONZÁLEZ, E. M. S. et al. Vegetación de la Sierra Madre Occidental, México: Una síntesis. Acta Botánica Mexicana. 100, 351-403, 2012b.

- GUJARATI, D. Econometría básica. D. F., México: Ed. McGrawHill/Interamericana, 463 pp., 1988.

- HARVEY, A. C. Estimating regression models with multiplicative heteroscedasticity. Econometrica, 44(3): 461-465, 1976.

- REYES VALDOVINOS, J. R. Sistema de cubicación para Eucaliptus grandis y E. urophylla en los límites de Veracruz y Oaxaca. Tesis profesional. Texcoco, Edo. de México, México: Universidad Autónoma de Chapingo. División de Ciencias Forestales, 36 pp., 2006.

- TORRES, R. J. M. y MAGAÑA, T. O. S. Evaluación de plantaciones forestales. D. F., México: Ed. Limusa Noriega, 473 pp., 2001.

- VARGAS, L. B. et al. Ecuaciones de volumen total y comercial para las especies maderables de la UMAFOR 1008 El Salto. Informe Técnico. 37 pp., 2012. 